TITLE:

\title{
Spectral curve and Hamiltonian structure of isomonodromic SU(2) Calogero-Gaudin system
}

\author{
$\operatorname{AUTHOR(S):~}$ \\ Takasaki, K
}

\section{CITATION:}

Takasaki, K. Spectral curve and Hamiltonian structure of isomonodromic SU(2) CalogeroGaudin system. JOURNAL OF MATHEMATICAL PHYSICS 2003, 44(9): 3979-3999

ISSUE DATE:

2003-09

URL:

http://hdl.handle.net/2433/50228

\section{RIGHT:}

Copyright 2003 American Institute of Physics. This article may be downloaded for personal use only. Any other use requires prior permission of the author and the American Institute of Physics.; この論文は出版社版でありません。引用 の際には出版社版をご確認ご利用ください。; This is not the published version. Please cite only the published version. 


\title{
Spectral curve and Hamiltonian structure of isomonodromic $\mathrm{SU}(2)$ Calogero-Gaudin system
}

\author{
Kanehisa Takasaki \\ Department of Fundamental Sciences \\ Faculty of Integrated Human Studies, Kyoto University \\ Yoshida, Sakyo-ku, Kyoto 606-8501, Japan \\ E-mail: takasaki@math.h.kyoto-u.ac.jp
}

\begin{abstract}
This paper presents a new approach to the Hamiltonian structure of isomonodromic deformations of a matrix system of ODE's on a torus. An isomonodromic analogue of the $\mathrm{SU}(2)$ Calogero-Gaudin system is used for a case study of this approach. A clue of this approach is a mapping to a finite number of points on the spectral curve of the isomonodromic Lax equation. The coordinates of these moving points give a new set of Darboux coordinates called the spectral Darboux coordinates. The system of isomonodromic deformations is thereby converted to a non-autonomous Hamiltonian system in the spectral Darboux coordinates. The Hamiltonians turn out to resemble those of a previously known isomonodromic system of a second order scalar ODE. The two isomonodromic systems are shown to be linked by a simple relation.
\end{abstract}

arXiv:nlin.SI/0111019 


\section{Comments on revised version}

This is a revised version of the paper published in Journal of Mathematical Physics vol. 44, no. 2 (2003), pp. 3979-3999. It turned out after publication that the published version contains serious errors on the construction of the Lax and zero-curvature equations. Namely, the $M$-matrices $M_{j}(z)$ have to be corrected by an extra diagonal matrix $\operatorname{diag}\left(p_{j},-p_{j}\right)$; the zero-curvature equations without these terms lead to a contradiction. The emergence of these correction terms was already pointed out in the paper of Korotkin and Samtleben [10]. As they stressed therein, this problem stems from the fact that the isomonodromic system in question is a constrained system. The quantities $p_{j}$, $j=1, \ldots, N$, may be interpreted as the Lagrange multipliers in the Hamiltonian formulation of this constrained system. This issue is also partly related to the treatment of the coefficient $\kappa$ that arises in the construction of spectral Darboux coordinates.

To correct these errors, Section III has been fully revised. Firstly, the "fake" Hamiltonians $\mathcal{H}_{j}$ are replaced by the Hamiltonians $\widetilde{\mathcal{H}}_{j}$ with a correction term proportional to the left hand side of the constraint. The equations of motion of the Calogero variables $q, p$ and the spin variables $A_{k}$ are defined by these Hamiltonians. The $t$-dependence of $p_{j}$ 's is not determined at this stage. Secondly, the $M$-matrices $M_{j}(z)$ are corrected by the diagonal matrix $\operatorname{diag}\left(p_{j},-p_{j}\right)$. The Lax and zero-curvature equations are reformulated in terms of the corrected $M$-matrices $\widetilde{M}_{j}(z)$. The diagonal part of the zero-curvature equations then yields a set of new differential equations for $p_{j}$ 's. As it turns out, the equations of motion of $q, p$ and $A_{k}$ 's ensure the integrability, in the sense of Frobenius, of these equations. Thus, as Korotkin and Samtleben [10] noted, the $t$-dependence of $p_{j}$ 's are eventually determined by the zero-curvature equations.

The subsequent sections are left mostly intact, except for the last part of Section VI that contained a wrong statement on $\kappa$.

I would like to express my gratitude to Henning Samtleben for helpful suggestions on this issue. 


\section{Introduction}

The notion of isomonodromic deformations originates in the celebrated work of R. Fuchs [1. Fuchs studied isomonodromic deformations of a second order linear ODE of the form

$$
\begin{aligned}
\frac{d^{2} y}{d z^{2}}+\left(\frac{a}{z^{2}}+\right. & \frac{b}{(z-1)^{2}}+\frac{c}{(z-t)^{2}}+\frac{d}{z(z-1)}-\frac{3}{4(z-\lambda)^{2}} \\
& \left.-\frac{t(t-1) K}{z(z-1)(z-t)}+\frac{\lambda(\lambda-1) \nu}{z(z-1)(z-\lambda)}\right) y=0
\end{aligned}
$$

with five regular singular points $z=0,1, \infty, t, \lambda$ on the Riemann sphere, and discovered a nonlinear ODE that is nowadays called the sixth Painlevé equation. His work was soon generalized by Garnier [2] and Schlesinger [3] in two different directions. Whereas Garnier extended the work of Fuchs to a second order linear ODE with more singularities (including irregular ones as well), Schlesinger studied a matrix system of the form

$$
\frac{d Y}{d z}=\sum_{j=1}^{N} \frac{A_{j}}{z-t_{j}} Y
$$

and obtained the so called Schlesinger system

$$
\frac{\partial A_{k}}{\partial t_{j}}=\left(1-\delta_{j k}\right) \frac{\left[A_{k}, A_{j}\right]}{t_{k}-t_{j}}-\delta_{j k} \sum_{l \neq k} \frac{\left[A_{k}, A_{l}\right]}{t_{k}-t_{l}}
$$

that characterizes isomonodromic deformations. It turned out afterwards 4] that Garnier's isomonodromic deformations with an arbitrary number of regular singular points can be reconstructed from the $2 \times 2$ Schlesinger system.

The next stage of generalization is, naturally, isomonodromic deformations on a torus. This issue was first tackled by Okamoto [5], who obtained a system of isomonodromic deformations of a second order scalar ODE on a torus. One of his remarkable results is that the isomonodromic system on a torus can be formulated as a Hamiltonian system in much the same way as Garnier's isomonodromic system on a sphere was converted to a Hamiltonian system [6]. Iwasaki [7] extended Okamoto's work to scalar ODE's of an arbitrary order on an arbitrary compact Riemann surface, and elucidated the geometric origin of the Hamiltonian structure that Okamoto derived. The study of isomonodromic systems on a torus was further refined by Okamoto himself [8] and Kawai [9].

As regards matrix systems, Korotkin and Samtleben [10] constructed an example of isomonodromic deformations of a $2 \times 2$ matrix system on a torus. Levin and Olshanetsky 
[1] developed a general framework in which the Schlesinger system and Korotkin and Samtleben's isomonodromic system are placed, along with generalizations to higher genus Riemann surfaces, in a unified way. Some more examples of matrix systems with different structures are also known [12, 13, 14, 15. Compared with Okamoto and Iwasaki's formulation, these "elliptic analogues of the Schlesinger system" are obtained on an entirely different ground, such as conformal field theories, vector bundles on a torus, KZ equations, and (classical or quantum) integrable systems. This can be seen in the structure of the matrix linear system

$$
\frac{d Y}{d z}=L(z) Y
$$

for which isomonodromic deformations are constructed. Namely, the matrix $L(z)$ (" $L$ matrix") in these examples is borrowed from the isospectral Lax equation of an integrable system, though the Lax equation of isomonodromic deformations takes the non-isospectral form

$$
\frac{\partial L(z)}{\partial t_{j}}=\left[L(z), M_{j}(z)\right]-\frac{\partial M_{j}(z)}{\partial z} .
$$

Each of those isomonodromic systems is thus accompanied by an isospectral partner.

The correspondence between isospectral and isomonodromic systems will have a number of significant implications. Among them, we are particularly interested in the role of "spectral Darboux coordinates". The notion of spectral Darboux coordinates was introduced by the Montreal group for isospectral systems with a rational $L$-matrix [16], and extended to isomonodromic systems on a sphere [17, 18. As they demonstrated for those cases, one can construct a mapping from the Lax equation of this type of systems to a dynamical system of a finite number of points $P_{1}, \ldots, P_{N}$ on the spectral curve

$$
\Gamma=\{(z, w) \mid \operatorname{det}(w I-L(z))=0\}
$$

though the spectral curve itself becomes dynamical in the case of isomonodromic deformations. Spectral Darboux coordinates are the coordinates $\lambda_{1}, \ldots, \lambda_{N}, \mu_{1}, \ldots, \mu_{N}$ of the moving points $P_{k}=\left(\lambda_{k}, \mu_{k}\right)$. These coordinates lead to "separation of variables" of isospectral systems. The most classical case is the so called Moser systems [19]; separation of variable of those systems was worked out by Moser himself. Remarkably, as Harnad and Wisse pointed out [18], almost the same story repeats on the isomonodromic side, 
except that separability is lost there. In particular, this shows an algebro-geometric interpretation of Okamoto's reformulation [6] of Garnier's work [4] on the $2 \times 2$ Schlesinger system.

This paper presents a similar approach to one of the "elliptic analogues" of the Schlesinger systems, namely, the aforementioned isomonodromic system of Korotkin and Samtleben (in a slightly modified form). The isospectral partner of this isomonodromic system is the Calogero-Gaudin system [20, 21] for the SU(2) group. Separation of variables of the usual SU(2) Calogero-Gaudin system has been developed by Brzeziński [22] and Enriquez et al. [23] (including "quantum separation of variables" in the sense of Sklyanin [24]). Our method is more or less parallel to theirs, in particular, that of Brzeziński. Actually, it is a rational (rather than elliptic) model of the SU(2) Calogero-Gaudin system that he considered. Thus we are to extend his method in two-fold ways - firstly, to an elliptic model (which is the subject of the work of Enriquez et al. as well), and secondly, to an isomonodromic system.

A main outcome of our consideration (summarized in Theorem 1, Section V) is that the isomonodromic SU(2) Calogero-Moser system can be converted to a non-autonomous Hamiltonian system in the spectral Darboux coordinates. The Hamiltonians of this nonautonomous system turn out to be a considerably intricate functions of the Darboux coordinates and the time variables. Remarkably, however, a very similar Hamiltonian system has been discovered by Okamoto [8] for isomonodromic deformations of a second order scalar ODE on a torus. We shall show a natural explanation of this coincidence from our pouint of view.

This paper is organized as follows. Sections II and III are for preparation. In Section II, the Poisson structure of the $L$-matrix of the Calogero-Gaudin systems is reviewed. In Section III, the isomonodromic system is formulated in terms of two canonically conjugate "Calogero variables" and a set of "spin variables". Section IV and V are the main part of this paper. In Section IV, the spectral curve and the spectral Darboux coordinates are introduced. In Section V, the non-autonomous Hamiltonian system is derived. Section VI deals with the relation to isomonodromic deformations of a second order scalar ODE. Section VII is for conclusion and supplementary remarks. Part of technical details are collected in Appendices. 


\section{II $\quad L$-matrix and Poisson structure}

\section{II.1 L-matrix}

Following the idea of Korotkin and Samtleben [10], we start from the $L$-matrix

$$
L(z)=\left(\begin{array}{cc}
p & 0 \\
0 & -p
\end{array}\right)+\sum_{j=1}^{N}\left(\begin{array}{cc}
\zeta\left(z-t_{j}\right) A_{j}^{3} & \phi\left(q, z-t_{j}\right) A_{j}^{-} \\
\phi\left(-q, z-t_{j}\right) A_{j}^{+} & -\zeta\left(z-t_{j}\right) A_{j}^{3}
\end{array}\right),
$$

where $q$ and $p$ are Calogero variables, $A_{j}^{ \pm}$and $A_{j}^{3}$ are spin variables, $\zeta(z)$ denotes the Weierstrass $\zeta$ function and $\phi(u, z)$ the auxiliary function that is widely used in the study of systems of the Calogero type:

$$
\zeta(z)=\frac{\sigma^{\prime}(z)}{\sigma(z)}, \quad \phi(u, z)=\frac{\sigma(u-z)}{\sigma(u) \sigma(z)} .
$$

Here $\sigma(z)$ is Weierstrass sigma function, and the prime stands for a derivative, i.e., $\sigma^{\prime}(z)=$ $d \sigma(z) / d z$. Let $2 \omega_{1}$ and $2 \omega_{3}$ denote the primitive periods of the Weierstrass functions. Throughout this paper, we assume that $t_{j} \neq t_{k}$ if $j \neq k$.

This $L$-matrix is slightly different from that of Korotkin and Samtleben [10]. They use Jacobi's elliptic theta function $\vartheta_{1}$ rather than Weierstrass' sigma function $\sigma$. Their $L$-matrix is thereby more suited for formulating isomonodromic deformations against the modulus $\tau$. We dare to modify Korotkin and Samtleben's $L$-matrix because this simplifies the use of interpolation formulae of elliptic functions. It should be possible to start from the $L$-matrix of Korotkin and Samtleben and to derive substantially the same results, though we shall not pursue it in this paper.

The Poisson structure of the dynamical variables is a standard one. The Calogero variables $q, p$ are, in fact, the relative coordinate $q_{1}-q_{2}$ and momentum $\left(p_{1}-p_{2}\right) / 2$ of a two body system with canonical variables $\left(q_{1}, q_{2}, p_{1}, p_{2}\right)$, and become a canonically

conjugate pair $\{q, p\}=1$ in themselves. The spin variables $A_{j}^{ \pm}, A_{j}^{3}$ obey the $\mathrm{su}(2)$ relations

$$
\left\{A_{j}^{3}, A_{k}^{ \pm}\right\}= \pm \delta_{j k} A_{k}^{ \pm}, \quad\left\{A_{j}^{+}, A_{k}^{-}\right\}=2 \delta_{j k} A_{k}^{3}
$$

with respect to the Poisson bracket.

The Poisson bracket of the spin variables is nothing but the Kostant-Killilov bracket for the residue matrix

$$
A_{j}=\left(\begin{array}{cc}
A_{j}^{3} & A_{j}^{-} \\
A_{j}^{+} & -A_{j}^{3}
\end{array}\right)
$$


of $L(z)$ at $z=t_{j}$. The conjugacy class

$$
\mathcal{O}_{j}=\left\{A_{j} \mid A_{j} \sim \operatorname{diag}\left(\theta_{j} / 2,-\theta_{j} / 2\right)\right\}
$$

of semi-simple matrices with fixed eigenvalues $\pm \theta_{j} / 2$ is a maximal (two-dimensional) symplectic leaf of this Poisson structure. One can use a canonically conjugate pair $\left(x_{j}, \xi_{j}\right)$, $\left\{x_{j}, \xi_{j}\right\}=1$, to parametrize this symplectic leaf as follows:

$$
A_{j}^{+}=-\frac{\xi_{j}^{2}}{2}+\frac{\theta_{j}^{2}}{2 x_{j}^{2}}, \quad A_{j}^{-}=\frac{x_{j}^{2}}{2}, \quad A_{j}^{3}=\frac{x_{j} \xi_{j}}{2} .
$$

Note that this parametrization is consistent with the Poisson bracket of $A_{j}^{ \pm, 3}$.

\section{II.2 Poisson bracket of $L$-matrix elements}

Let us write the matrix elements of $L(z)$ as

$$
L(z)=\left(\begin{array}{cc}
A(u) & B(u) \\
C(u) & -A(u)
\end{array}\right)
$$

More explicitly,

$$
\begin{aligned}
& A(u)=p+\sum_{j=1}^{N} \zeta\left(z-t_{j}\right) A_{j}^{3}, \\
& B(u)=\sum_{j=1}^{N} \phi\left(q, z-t_{j}\right) A_{j}^{-}, \\
& C(u)=\sum_{j=1}^{N} \phi\left(-q, z-t_{j}\right) A_{j}^{+} .
\end{aligned}
$$

The non-zero Poisson brackets of these matrix elements take the form

$$
\begin{aligned}
& \{A(z), B(w)\}=B(z) \phi(-q, z-w)-B(w) \zeta(z-w) \\
& \{A(z), C(w)\}=-C(z) \phi(q, z-w)+C(w) \zeta(z-w) \\
& \{B(z), C(w)\}=2(A(z)-A(w)) \phi(q, z-w)+2 \phi_{u}(q, z-w) \sum_{j=1}^{N} A_{j}^{3} .
\end{aligned}
$$

where

$$
\phi_{u}(u, z)=\frac{\partial \phi(u, z)}{\partial u}=-\phi(u, z)(\zeta(z-u)+\zeta(u)) .
$$


Thus the Poisson algebra of the matrix elements of $L(z)$ almost closes up to the extra term proportional to $\sum_{j=1}^{N} A_{j}^{3}$, which is later set to zero in order to derive the Lax equation.

These Poisson commutation relations can be easily verified by direct calculations using the functional identity

$$
\phi(u, z) \phi(-u, w)+\phi(u, z-w)(\zeta(z)-\zeta(w))+\phi_{u}(u, z-w)=0
$$

of the auxiliary functions. This functional identity is a consequence of the more general one

$$
\phi(u, z) \phi(v, w)+\phi(u+v, z) \phi(-v, z-w)-\phi(u+v, w) \phi(u, z-w)=0,
$$

from which the former identity can be derived by letting $v \rightarrow-u$.

The Poisson structure of the $L(z)$-matrix elements can be cast into the compact form

$$
\begin{aligned}
\{L(z) \stackrel{\otimes}{, L} L(w)\} & =\sum_{a, b, c, d}\left\{L_{a b}(z), L_{c d}(w)\right\} E_{a b} \otimes E_{c d} \\
& =[L(z) \otimes I+I \otimes L(w), r(z-w)]+2 \frac{\partial r(z-w)}{\partial q} \sum_{j=1}^{N} A_{j}^{3}
\end{aligned}
$$

where $E_{a b}$ denotes the matrix with the $(a, b)$ element equal to 1 and the other elements vanishing. The $r$-matrix takes the form

$$
\begin{aligned}
r(z-w)= & \zeta(z-w) E_{11} \otimes E_{11}+\phi(q, z-w) E_{12} \otimes E_{21} \\
& +\phi(-q, z-w) E_{21} \otimes E_{12}+\zeta(z-w) E_{22} \otimes E_{22} \\
= & \left(\begin{array}{cccc}
\zeta(z-w) & 0 & 0 & 0 \\
0 & 0 & \phi(q, z-w) & 0 \\
0 & \phi(-q, z-w) & 0 & 0 \\
0 & 0 & 0 & \zeta(z-w)
\end{array}\right),
\end{aligned}
$$

which is a special case of the well known dynamical $r$-matrix of the elliptic Calogero-Moser system [25, 26, 27. 


\section{Hamiltonians and Lax equations}

\section{III.1 Hamiltonians}

The fundamental Poisson commutation relation (13) implies that the standard quadratic Hamiltonians

$$
\mathcal{H}_{j}=\operatorname{Res}_{z=t_{j}} \frac{1}{2} \operatorname{Tr} L(z)^{2}
$$

are not Poisson-commutative in themselves, but commute up to a term proportional to $\sum_{j=1}^{N} A_{j}^{3}$ :

$$
\left\{\mathcal{H}_{j}, \mathcal{H}_{k}\right\} \propto \sum_{j=1}^{N} A_{j}^{3}
$$

The factor $\sum_{j=1}^{N} A_{j}^{3}$ itself commutes with $\mathcal{H}_{j}$ 's:

$$
\left\{\sum_{j=1}^{N} A_{j}^{3}, \mathcal{H}_{k}\right\}=0
$$

To obtain a commuting set of flows, therefore, one has to impose the constraint

$$
\sum_{j=1}^{N} A_{j}^{3}=0
$$

Note that $\sum_{j=1}^{N} A_{j}^{3}$ is an infinitesimal generator of the diagonal gauge transformations

$$
A_{j} \mapsto g^{-1} A_{j} g, \quad g=\left(\begin{array}{cc}
e^{\gamma} & 0 \\
0 & -e^{\gamma}
\end{array}\right)
$$

Because of the presence of the constraint (18), as Korotkin and Samtleben [10] pointed out, one has to modify the naive Hamiltonians $\mathcal{H}_{j}$ by a term proportional to the left hand side of the constraint as

$$
\widetilde{\mathcal{H}}_{j}=\mathcal{H}_{j}-2 p_{j} \sum_{k=1}^{N} A_{k}^{3}
$$

The multipliers $p_{j}, j=1, \ldots, N$, are assumed to satisfy the relation

$$
\sum_{j=1}^{N} p_{j}=p
$$


which ensures the consistency of Lax and zero-curvature equations that we shall derive later on. A set of commuting flows are now defined on the reduced phase space by the canonical equations

$$
\frac{\partial q}{\partial t_{j}}=\left\{q, \widetilde{\mathcal{H}}_{j}\right\}, \quad \frac{\partial p}{\partial t_{j}}=\left\{p, \widetilde{\mathcal{H}}_{j}\right\}, \quad \frac{\partial A_{k}}{\partial t_{j}}=\left\{A_{k}, \widetilde{\mathcal{H}}_{j}\right\}
$$

The Poisson brackets on the right hand side are understood to be calculated as

$$
\left\{F, \widetilde{\mathcal{H}}_{j}\right\}=\left\{F, \mathcal{H}_{j}\right\}-2 p_{j}\left\{F, \sum_{j=1}^{N} A_{j}\right\} .
$$

Namely, we first calculate the Poisson bracket in the unconstrained variables, then impose the constraint. Since the term containing $\left\{F, p_{j}\right\}$ disappears upon imposing the constraint, we leave the Poisson brackets with $p_{j}$ 's undefined. More explicitly, the equations of motion read as follows.

1. Equations of motion of $q$ and $p$ :

$$
\begin{aligned}
\frac{\partial q}{\partial t_{j}} & =2 A_{j}^{3} \\
\frac{\partial p}{\partial t_{j}} & =-\sum_{k \neq j} \phi_{u}\left(q, t_{j}-t_{k}\right) A_{j}^{+} A_{l}^{-}+\sum_{k \neq j} \phi_{u}\left(-q, t_{j}-t_{k}\right) A_{j}^{-} A_{k}^{+} .
\end{aligned}
$$

2. Equations of motion of $A_{k}^{ \pm}$:

$$
\begin{aligned}
\frac{\partial A_{k}^{ \pm}}{\partial t_{j}} & =\mp 2 \zeta\left(t_{j}-t_{k}\right) A_{j}^{3} A_{k}^{ \pm} \pm 2 \phi\left( \pm q, t_{j}-t_{k}\right) A_{j}^{ \pm} A_{k}^{3} \pm 2 p_{j} A_{k}^{ \pm} \quad(j \neq k), \\
\frac{\partial A_{k}^{ \pm}}{\partial t_{k}} & =\mp 2 \sum_{\ell \neq k} \zeta\left(t_{k}-t_{\ell}\right) A_{k}^{ \pm} A_{\ell}^{3} \pm 2 \sum_{\ell \neq k} \phi\left(\mp q, t_{k}-t_{\ell}\right) A_{k}^{3} A_{\ell}^{ \pm} \mp 2 \sum_{\ell \neq k} p_{\ell} A_{k}^{ \pm} .
\end{aligned}
$$

3. Equations of motion of $A_{k}^{3}$ :

$$
\begin{aligned}
\frac{\partial A_{k}^{3}}{\partial t_{j}} & =-\phi\left(q, t_{j}-t_{k}\right) A_{j}^{+} A_{k}^{-}+\phi\left(-q, t_{j}-t_{k}\right) A_{j}^{-} A_{k}^{+} \quad(j \neq k), \\
\frac{\partial A_{k}^{3}}{\partial t_{k}} & =\sum_{\ell \neq k} \phi\left(q, t_{k}-t_{\ell}\right) A_{k}^{+} A_{\ell}^{-}-\sum_{\ell \neq k} \phi\left(-q, t_{k}-t_{\ell}\right) A_{k}^{-} A_{\ell}^{+} .
\end{aligned}
$$

In particular, the sum of these flows turns out to be trivial:

$$
\sum_{j=1}^{N} \frac{\partial q}{\partial t_{j}}=0, \quad \sum_{j=1}^{N} \frac{\partial p}{\partial t_{j}}=0, \quad \sum_{j=1}^{N} \frac{\partial A_{k}}{\partial t_{j}}=0 .
$$

The $t$-dependence of $p_{j}$ 's cannot be determined in this way; we shall derive a set of differential equations for $p_{j}$ 's later on. 


\section{III.2 Calculating $\left\{L(z), \widetilde{\mathcal{H}}_{j}\right\}$}

As an intermediate step towards Lax equations, we now consider the Poisson bracket

$$
\left\{L(z), \widetilde{\mathcal{H}}_{j}\right\}=\left\{L(z), \mathcal{H}_{j}\right\}-2 p_{j}\left\{L(z), \sum_{k=1}^{N} A_{k}^{3}\right\}
$$

of $L(z)$ with the modified Hamiltonians $\widetilde{H}_{j}$. A clue is the general formula

$$
\left\{L(z), \frac{1}{n} \operatorname{Tr} L(w)^{n}\right\}=\operatorname{Tr}_{2}\left(\{L(z) \stackrel{\otimes}{,} L(w)\} I \otimes L(w)^{n-1}\right),
$$

where $\operatorname{Tr}_{2}$ denotes the trace over the second component of the tensor product:

$$
\operatorname{Tr}_{2}\left(\sum_{a, b, c, d} X_{a b c d} E_{a b} \otimes E_{c d}\right)=\sum_{a, b}\left(\sum_{c} X_{a b c c}\right) E_{a b}
$$

Plugging the Poisson commutation relation (13) into this formula, one obtains the identity

$$
\begin{aligned}
\left\{L(z), \frac{1}{2} \operatorname{Tr} L(w)^{2}\right\}= & {\left[L(z), \operatorname{Tr}_{2}(r(z-w) I \otimes L(w))\right] } \\
& +2 \operatorname{Tr}_{2}\left(\frac{\partial r(z-w)}{\partial q} I \otimes L(w)\right) \sum_{j=1}^{N} A_{j}^{3} .
\end{aligned}
$$

Extracting the residues at $w=t_{j}$ yields the Poisson bracket $\left\{L(z), \mathcal{H}_{j}\right\}$. Note that the second term on the right hand side disappears upon imposing the constraint. Since

$$
\operatorname{Res}_{w=t_{j}} \operatorname{Tr}_{2}(r(z-w) I \otimes L(w))=\left(\begin{array}{cc}
\zeta\left(z-t_{j}\right) A_{j}^{3} & \phi\left(q, z-t_{j}\right) A_{j}^{-} \\
\phi\left(-q, z-t_{j}\right) A_{j}^{+} & -\zeta\left(z-t_{j}\right) A_{j}^{3}
\end{array}\right),
$$

the Poisson bracket with $\mathcal{H}_{j}$ eventually takes the form

$$
\left\{L(z), \mathcal{H}_{j}\right\}=\left[L(z), M_{j}(z)\right]
$$

where

$$
M_{j}(z)=\left(\begin{array}{cc}
\zeta\left(z-t_{j}\right) A_{j}^{3} & \phi\left(q, z-t_{j}\right) A_{j}^{-} \\
\phi\left(-q, z-t_{j}\right) A_{j}^{+} & -\zeta\left(z-t_{j}\right) A_{j}^{3}
\end{array}\right) .
$$

On the other hand, the Poisson bracket with $\sum_{k=1}^{N} A_{k}^{3}$ can be readily calculated as

$$
\begin{aligned}
\left\{L(z), \sum_{k=1}^{N} A_{k}^{3}\right\} & =\sum_{k=1}^{N}\left(\begin{array}{cc}
0 & \phi\left(q, z-t_{k}\right) A_{k}^{-} \\
\phi\left(-q, z-t_{k}\right) A_{k}^{+} & 0
\end{array}\right) \\
& =[L(z), \operatorname{diag}(-1 / 2,1 / 2)] .
\end{aligned}
$$


One thus finds that

$$
\left\{L(z), \widetilde{\mathcal{H}}_{j}\right\}=\left[L(z), \widetilde{M}_{j}(z)\right]
$$

where

$$
\widetilde{M}_{j}(z)=M_{j}(z)+\operatorname{diag}\left(p_{j},-p_{j}\right)
$$

\section{III.3 Lax equations}

We are now ready to derive the Lax equations.

By the Leibniz rule, the $t$-derivatives of the matrix elements of $L(z)$ can be written as

$$
\begin{array}{r}
\frac{\partial L_{12}(z)}{\partial t_{j}}=\sum_{k=1}^{N}\left(\phi_{u}\left(q, z-t_{k}\right) \frac{\partial q}{\partial t_{j}} A_{k}^{-}+\phi\left(q, z-t_{k}\right) \frac{\partial A_{k}^{-}}{\partial t_{j}}\right)-\phi^{\prime}\left(q, z-t_{j}\right) A_{j}^{-}, \\
\frac{\partial L_{21}(z)}{\partial t_{j}}=\sum_{k=1}^{N}\left(-\phi_{u}\left(-q, z-t_{j}\right) \frac{\partial q}{\partial t_{j}} A_{k}^{+}+\phi\left(-q, z-t_{j}\right) \frac{\partial A_{k}^{+}}{\partial t_{j}}\right)-\phi^{\prime}\left(-q, z-t_{j}\right) A_{j}^{+},
\end{array}
$$

and

$$
\frac{\partial L_{11}(z)}{\partial t_{j}}=-\frac{\partial L_{22}(z)}{\partial t_{j}}=\frac{\partial p}{\partial t_{j}}+\sum_{k=1}^{N} \zeta\left(z-t_{k}\right) \frac{\partial A_{k}^{3}}{\partial t_{j}}-\zeta^{\prime}\left(z-t_{j}\right) A_{j}^{3}
$$

where

$$
\phi^{\prime}(u, z)=\frac{\partial \phi(q, z)}{\partial z}=-\phi(u, z)(\zeta(u-z)+\zeta(z))
$$

Notice here that the terms containing $\phi^{\prime}\left( \pm q, z-t_{j}\right)$ and $\zeta\left(z-t_{j}\right)$ coincide with the matrix elements of $-\partial \widetilde{M}_{j}(z) / \partial z$. On the other hand, the $t_{j}$-derivatives of the dynamical variables $q, p, A_{k}$ can be expressed as the Poisson bracket with $\widetilde{\mathcal{H}}_{j}$. Consequently,

$$
\frac{\partial L(z)}{\partial t_{j}}=\left\{L(z), \mathcal{H}_{j}\right\}-\frac{\partial \widetilde{M}_{j}(z)}{\partial z} .
$$

Combining this with the foregoing calculation of $\left\{L(z), \mathcal{H}_{j}\right\}$, we eventually obtain the Lax equations

$$
\frac{\partial L(z)}{\partial t_{j}}=\left[L(z), \widetilde{M}_{j}(z)\right]-\frac{\partial \widetilde{M}_{j}(z)}{\partial z}
$$

of the isomonodromic type. Though we omit details, one can conversely derive the equations of motion of $q, p$ and $A_{k}$ 's from these Lax equations. 
As a remark, let us mention that these Lax equations are consistent with (27). This is a consequence of the linear relation (21) among $p_{j}$ 's and $p$. One can derive, from this linear relation, the linear relation

$$
\sum_{j=1}^{N} \widetilde{M}_{j}(z)=L(z)
$$

among the matrices $\widetilde{M}_{j}(z)$ and $L(z)$. The sum of the $N$ Lax equations thereby yields the relation

$$
\sum_{j=1}^{N} \frac{\partial L(z)}{\partial t_{j}}=-\frac{\partial L(z)}{\partial z}
$$

which is a restatement of (27).

\section{III.4 Zero-curvature equations}

The Lax equations are self-consistent (i.e., define commutating flows) if and only if the zero-curvature equations

$$
\frac{\partial \widetilde{M}_{k}(z)}{\partial t_{j}}-\frac{\partial \widetilde{M}_{j}(z)}{\partial t_{k}}+\left[\widetilde{M}_{j}(z), \widetilde{M}_{k}(z)\right]=0
$$

are satisfied. As we show below, these equations give a set of differential equations for $p_{j}$ 's.

The differential equations for $p_{j}$ 's are obtained from the the diagonal part of the zerocurvature equations. We have only to consider the upper left component, because all matrices in the zero-curvature equations are trace-free. The diagonal part $\left[\widetilde{M}_{j}(z), \widetilde{M}_{k}(z)\right]_{11}=$ $-\left[\widetilde{M}_{j}(z), \widetilde{M}_{k}(z)\right]_{22}$ of the commutator can be rewritten as

$$
\begin{aligned}
{\left[\widetilde{M}_{j}(z), \widetilde{M}_{k}(z)\right]_{11}=} & \phi\left(q, z-t_{j}\right) \phi\left(-q, z-t_{k}\right) A_{j}^{-} A_{k}^{+}-\phi\left(q, z-t_{k}\right) \phi\left(-q, z-t_{j}\right) A_{k}^{-} A_{j}^{+} \\
= & -\left(\phi\left(q, t_{k}-t_{j}\right)\left(\zeta\left(z-t_{j}\right)-\zeta\left(z-t_{k}\right)\right)+\phi_{u}\left(q, t_{k}-t_{j}\right)\right) A_{j}^{-} A_{k}^{+} \\
& +\left(\phi\left(q, t_{j}-t_{k}\right)\left(\zeta\left(z-t_{k}\right)-\zeta\left(z-t_{j}\right)\right)+\phi_{u}\left(q, t_{j}-t_{k}\right)\right) A_{k}^{-} A_{j}^{+} .
\end{aligned}
$$

The functional identity (11) has been used in the last stage. The $z$-dependent pieces are thus a linear combination of $\zeta\left(z-t_{j}\right)$ and $\zeta\left(z-t_{k}\right)$. On the other hand, the derivative part $\partial \widetilde{M}_{k, 11}(z) / \partial t_{j}-\partial \widetilde{M}_{j, 11}(z) / \partial t_{k}$ of the zero-curvature equation can be expressed as

$$
\frac{\partial \widetilde{M}_{k, 11}(z)}{\partial t_{j}}-\frac{\partial \widetilde{M}_{j, 11}(z)}{\partial t_{k}}=\frac{\partial p_{k}}{\partial t_{j}}+\zeta\left(z-t_{k}\right) \frac{\partial A_{k}^{3}}{\partial t_{j}}-\frac{\partial p_{j}}{\partial t_{k}}-\zeta\left(z-t_{j}\right) \frac{\partial A_{j}^{3}}{\partial t_{k}} .
$$


Upon summation with the foregoing expression of the commutator part, the $z$-dependent pieces $\zeta\left(z-t_{j}\right)(\cdots)+\zeta\left(z-t_{k}\right)(\cdots)$ turn out to cancel out as a consequence of the equations of motion of $A_{j}^{3}$ and $A_{k}^{3}$. One is thus left with the differential equations

$$
\frac{\partial p_{k}}{\partial t_{j}}-\frac{\partial p_{j}}{\partial t_{k}}+\phi_{u}\left(q, t_{j}-t_{k}\right) A_{j}^{+} A_{k}^{-}-\phi_{u}\left(q, t_{k}-t_{j}\right) A_{j}^{-} A_{k}^{+}=0
$$

for $p_{j}$ 's.

One can examine the off-diagonal part of the zero-curvature equations in much the same way. This however leads to no new equations (see Appendix C). Namely, all equations are satisfied under the equations of motion of $q, p$ and $A_{j}$ 's.

The final problem is the existence of a solution of (38). To this end, it is convenient to convert (38) to an exterior differential equation. Let $\theta$ denote the one-form

$$
\theta=\sum_{j=1}^{N} p_{j} d t_{j} .
$$

(38) thereby turns into the exterior differential equation

$$
d \theta=\omega,
$$

where

$$
\begin{aligned}
\omega & =-\frac{1}{2} \sum_{j, k=1}^{N} \phi_{u}\left(q, t_{j}-t_{k}\right) A_{j}^{+} A_{k}^{-}+\frac{1}{2} \sum_{j, k=1}^{N} \phi_{u}\left(q, t_{k}-t_{j}\right) A_{j}^{-} A_{k}^{+} \\
& =\sum_{j, k=1}^{N} \phi\left(q, t_{j}-t_{k}\right)\left(\zeta\left(t_{j}-t_{k}-q\right)+\zeta(q)\right) A_{j}^{+} A_{k}^{-} .
\end{aligned}
$$

Consequently, the Frobenius integrability of (38) is equivalent to the closedness

$$
d \omega=0
$$

of $\omega$. Actually, this integrability condition turns out to be satisfied under the equations of motion of $q, p$ and $A_{j}$ (see Appendix D).

\section{Spectral curve and Darboux coordinates}

\section{IV.1 Spectral curve}

The spectral curve is defined by the eigenvalue equation

$$
\operatorname{det}(w I-L(z))=0
$$


Since $L(z)$ is trace-free, the left hand side can be rewritten as

$$
\operatorname{det}(w I-L(z))=w^{2}+\operatorname{det} L(z)=w^{2}-\frac{1}{2} \operatorname{Tr} L(z)^{2} .
$$

Under the constraint (18), the matrix elements of $L(z)$ enjoy the following quasiperiodicity along the period lattice of the torus:

$$
L\left(z+2 m \omega_{1}+2 n \omega_{3}\right)=e^{-\left(m \eta_{1}+n \eta_{3}\right) Q} L(z) e^{\left(m \eta_{1}+n \eta_{3}\right) Q},
$$

where $Q$ is the diagonal matrix $Q=\operatorname{diag}(q,-q)$, and $\eta_{1}$ and $\eta_{3}$ are the values of $\zeta(z)$ at $z=\omega_{1}, \omega_{3}$. The quasi-periodicity of $L(z)$ is a consequence of the quasi-periodicity of $\zeta(z)$ and $\phi(u, z)$,

$$
\begin{aligned}
\zeta\left(z+2 m \omega_{1}+2 n \omega_{3}\right) & =\zeta(z)+2 m \eta_{1}+2 n \omega_{3}, \\
\phi\left(u, z+2 m \omega_{1}+2 m \omega_{3}\right) & =e^{-2 m \eta_{1}-2 n \eta_{3}} \phi(u, z),
\end{aligned}
$$

which are easy to confirm from the property of the sigma function.

The quasi-periodicity of $L(z)$, in particular, implies the double periodicity of $\operatorname{Tr} L(z)^{2} / 2$, which thereby becomes an elliptic function with poles at $z=t_{1}, \ldots, t_{N}$. Since

$$
L(z)=\frac{A_{j}}{z-t_{j}}+O(1)
$$

as $z \rightarrow t_{j}$, this elliptic function has a double pole at $z=t_{j}$ with the leading coefficient equal to the quadratic Casimir

$$
C_{j}=\frac{1}{2} \operatorname{Tr} A_{j}^{2}=\frac{\theta_{j}^{2}}{4}
$$

of $A_{j}$. The residue is nothing but the Hamiltonian $\mathcal{H}_{j}$. Thus $\operatorname{Tr} L(z)^{2} / 2$ can be expressed as

$$
\frac{1}{2} \operatorname{Tr} L(z)^{2}=\sum_{j=1}^{N} C_{j} \wp\left(z-t_{j}\right)+\sum_{j=1}^{N} \mathcal{H}_{j} \zeta\left(z-t_{j}\right)+\mathcal{H}_{0}
$$

where $\mathcal{H}_{0}$ is a constant term (which however depends on $\omega_{1}$ and $\omega_{3}$ ). Also note that the Hamiltonians are not linearly independent, but obey the linear constraint

$$
\sum_{j=1}^{N} \mathcal{H}_{j}=0
$$


This is a consequence of the the double periodicity of $\operatorname{Tr} L(z)^{2} / 2$.

The spectral curve thus turns out to be a double covering of the torus. The branch points are located above the (possibly multiple) $2 N$ zeros of $\operatorname{Tr} L(z)^{2} / 2$. If these zeros are all simple, the genus of the spectral curve is equal to $N+1$. The spectral curve is time-dependent because of the extra term $\partial M_{j}(z) / \partial z$ on the right hand side of the Lax equation.

\section{IV.2 Spectral Darboux coordinates}

The construction of spectral Darboux coordinates is parallel to the case of the rational (and isospectral) model [22]. The "coordinate part" $\lambda_{1}, \ldots, \lambda_{N}$ are defined as the $N$ zeros (modulo the period lattice) of $L_{12}(z)$,

$$
L_{12}\left(\lambda_{j}\right)=0
$$

and the "momentum part" $\mu_{1}, \ldots, \mu_{N}$ are defined to be the value of $L_{11}(z)$ at these points,

$$
\mu_{j}=L_{11}\left(\lambda_{j}\right)=p+\sum_{k=1}^{N} \zeta\left(\lambda_{j}-t_{k}\right) A_{k}^{3} .
$$

In order to avoid a delicate problem, we assume throughout the following consideration

that $\lambda_{j} \neq \lambda_{k}$ if $j \neq k$. It is easy to see that $\left(\lambda_{j}, \mu_{j}\right)$ sits on the spectral curve; $L\left(\lambda_{j}\right)$ takes the triangular form

$$
L\left(\lambda_{j}\right)=\left(\begin{array}{cc}
\mu_{k} & 0 \\
L_{21}\left(\lambda_{j}\right) & -\mu_{k}
\end{array}\right)
$$

which implies that $\pm \mu_{j}$ are eigenvalues of $L\left(\lambda_{j}\right)$.

The $\lambda_{j}$ 's are constrained by a linear relation. To see this, let us note that $L_{12}(z)$ can be factorized as

$$
L_{12}(z)=\kappa \frac{\prod_{j=1}^{N} \sigma\left(z-\lambda_{j}\right)}{\prod_{j=1}^{N} \sigma\left(z-t_{j}\right)},
$$

where $\kappa$ is a constant that does not depend on $z$. The quasi-periodicity

$$
L_{12}\left(z+2 m \omega_{1}+2 n \omega_{3}\right)=e^{-\left(2 m \eta_{1}+2 n \eta_{3}\right) q} L_{12}(z)
$$


of $L_{12}(z)$ implies that its zeros $\lambda_{1}, \ldots, \lambda_{N}$ are constrained as

$$
\sum_{j=1}^{N} \lambda_{j}-\sum_{j=1}^{N} t_{j} \equiv q \quad \bmod 2 \omega_{1} \mathbf{Z}+2 \omega_{3} \mathbf{Z} .
$$

Since each $\lambda_{j}$ is defined only up to a difference by an element of the period lattice, let us redefine $\lambda_{j}$ 's, if necessary, such that this holds without "mod $2 \omega_{1} \mathbf{Z}+2 \omega_{3} \mathbf{Z}$ ":

$$
\sum_{j=1}^{N} \lambda_{j}-\sum_{j=1}^{N} t_{j}=q
$$

Of course this will be valid only for a local study of the system; this naive prescription has to be modified if one considers a global problem.

Let us note here that the coefficient $\kappa$ transforms as $\kappa \rightarrow e^{2 \gamma} \kappa$ under diagonal gauge transformation (19). One can thereby adjust $\kappa$ to any non-zero value, e.g.,

$$
\kappa=1
$$

In other words, $\kappa$ is not a true dynamical degree of freedom.

\section{IV.3 Time-dependent canonical transformation}

In order to prove the canonicity of these variables $\lambda_{j}, \mu_{j}$, we now restrict the spin variables onto the direct product $\mathcal{O}_{1} \times \cdots \times \mathcal{O}_{N}$ of the symplectic leaves and use the parametrization (6) by $\left(x_{j}, \xi_{j}\right)$. Moreover, we tentatively relax the constraint (18), which now takes the form

$$
\sum_{j=1}^{N} x_{j} \xi_{j}=0
$$

and restore it in the final stage.

The factorization relation (53) of $L_{12}(z)$ now reads

$$
\frac{1}{2} \sum_{j=1}^{N} \phi\left(q, z-t_{j}\right) x_{j}^{2}=\kappa \frac{Q(z)}{P(z)}
$$

where we have introduced the two functions

$$
Q(z)=\prod_{j=1}^{N} \sigma\left(z-\lambda_{j}\right), \quad P(z)=\prod_{j=1}^{N} \sigma\left(z-t_{j}\right) .
$$


This reduces to the relations

$$
\frac{1}{2} x_{j}^{2}=\kappa \frac{Q\left(t_{j}\right)}{P^{\prime}\left(t_{j}\right)}=\kappa \frac{\prod_{k=1}^{N} \sigma\left(t_{j}-\lambda_{k}\right)}{\prod_{k \neq j} \sigma\left(t_{j}-t_{k}\right)}
$$

of the residues of both hand sides at $z=t_{j}$. These relations show how the old variables $x_{j}$ are connected with the new variables $\lambda_{j}$ (and $\left.\kappa\right)$. By logarithmic differentiation, these relations can be further converted to the linear relations

$$
\begin{aligned}
2 d \log x_{j} & =d \log \kappa+\sum_{k=1}^{N} d \log \sigma\left(t_{j}-\lambda_{k}\right)-\sum_{k \neq j} d \log \sigma\left(t_{j}-t_{k}\right) \\
& =d \log \kappa+\sum_{k=1}^{N} \zeta\left(t_{j}-\lambda_{k}\right)\left(d t_{j}-d \lambda_{k}\right)-\sum_{k \neq j} \zeta\left(t_{j}-t_{k}\right)\left(d t_{j}-d t_{k}\right) .
\end{aligned}
$$

of differential forms.

Our goal is to derive a relation between the canonical one-forms $\sum_{j=1}^{N} \xi_{j} d x_{j}+p d q$ and $\sum_{j=1}^{N} \mu_{j} d \lambda_{j}$. We first multiply the both hand sides of the last relation by $x_{j} \xi_{j} / 2$, sum over $j=1, \ldots, N$, and add $p d q$ to both hand sides. We then obtain the linear relation

$$
\begin{aligned}
\sum_{j=1}^{N} \xi_{j} d x_{j}+p d q= & \frac{1}{2} \sum_{j=1}^{N} x_{j} \xi_{j} d \log \kappa+\frac{1}{2} \sum_{j, k=1}^{N} x_{j} \xi_{j} \zeta\left(t_{j}-\lambda_{k}\right)\left(d t_{j}-d \lambda_{k}\right) \\
& -\frac{1}{2} \sum_{j \neq k} x_{j} \xi_{j} \zeta\left(t_{j}-t_{k}\right)\left(d t_{j}-d t_{k}\right)+p d q
\end{aligned}
$$

On the other hand, by differentiating (55), we have the relation

$$
d q=\sum_{j=1}^{N} d \lambda_{j}-\sum_{j=1}^{N} d t_{j}
$$

which we can use to eliminate the differential $d q$ on the right hand side of the foregoing linear relation of one-forms. The right hand side thereby becomes a linear combination of $d \log \kappa, d \lambda_{j}$ 's and $d t_{j}$ 's, and the coefficient of $d \lambda_{j}$ turns out to be equal to $\mu_{j}$ by (52). We thus eventually find that

$$
\begin{aligned}
\sum_{j=1}^{N} \xi_{j} d x_{j}+p d q= & \frac{1}{2} \sum_{j=1}^{N} x_{j} \xi_{j} d \log \kappa+\sum_{j=1}^{N} \mu_{j} d \lambda_{j} \\
& -p \sum_{j=1}^{N} d t_{j}+\frac{1}{2} \sum_{j, k=1}^{N} x_{j} \xi_{j} \zeta\left(t_{j}-\lambda_{k}\right) d t_{j} \\
& -\frac{1}{2} \sum_{j \neq k} x_{j} \xi_{j} \zeta\left(t_{j}-t_{k}\right)\left(d t_{j}-d t_{k}\right) .
\end{aligned}
$$


The last equation shows that $\lambda_{j}$ and $\mu_{j}$ are Darboux coordinates of the canonical one-form $\sum_{j=1}^{N} \xi_{j} d x_{j}+p d q$, and that $\log \kappa$ is a conjugate variable of the left hand side of constraint (57). This interpretation is fully parallel to the spectral description of rational isospectral systems [16, 17, 18].

An essential difference lies in the fact that the time variables explicitly enter the relation between the two canonical one-forms. This means that the spectral Darboux coordinates are connected with the old variables $\left(x_{j}, \xi_{j}, q, p\right)$ by a time-dependent canonical transformation. Accordingly, the Hamiltonians (which is denoted by $H_{j}$ in the following) in the spectral Darboux coordinates differ from the Hamiltonians $\mathcal{H}_{j}$ in the old variables $\left(x_{j}, \xi_{j}, q, p\right)$. Their relation is to be determined by the fundamental formula

$$
\sum_{j=1}^{N} \xi_{j} d x_{j}+p d q-\sum_{j=1}^{N} \mathcal{H}_{j} d t_{j}=\sum_{j=1}^{N} \mu_{j} d \lambda_{j}-\sum_{j=1}^{N} H_{j} d t_{j}
$$

in which we have imposed the constraint (57). More explicitly, the new Hamiltonians are defined as

$$
H_{j}=\mathcal{H}_{j}+p-\frac{1}{2} x_{j} \xi_{j}\left(\sum_{k=1}^{N} \zeta\left(t_{j}-\lambda_{k}\right)-\sum_{k \neq j} \zeta\left(t_{j}-t_{k}\right)\right)+\frac{1}{2} \sum_{k \neq j} \zeta\left(t_{j}-t_{k}\right) x_{k} \xi_{k} .
$$

The goal of the next section is to rewrite the right hand side in terms of the spectral Darboux coordinates.

\section{Hamiltonian system in spectral Darboux coordi- nates}

\section{V.1 Linear equations characterizing Hamiltonians}

Let us recall that the pairs $\left(\lambda_{j}, \mu_{j}\right)$ of the spectral Darboux coordinates all sit on the spectral curve. Therefore the equations

$$
\mu_{k}^{2}=\sum_{j=1}^{N} C_{j} \wp\left(\lambda_{k}-t_{j}\right)+\sum_{j=1}^{N} \mathcal{H}_{j} \zeta\left(\lambda_{k}-t_{j}\right)+\mathcal{H}_{0}
$$

are satisfied for $k=1, \ldots, N$. These equations, along with the linear constraint (50), may be thought of as a system of linear equations that determine $\mathcal{H}_{j}$ 's. In fact, as we shall 
discuss afterwards, these linear equations can be solved for $\mathcal{H}_{j}$ 's, which thereby becomes an explicit function of the spectral Darboux coordinates (and of the time variables).

If the system in consideration were an isospectral system (such as the Moser system or the usual Calogero-Gaudin system), the time variables would not appear here explicitly, and $\mathcal{H}_{j}$ 's would be the Hamiltonians that we have sought. The only problem would have been to solve the foregoing linear equations for $\mathcal{H}_{j}$. This is, actually, what Brzeziński 22] and Enriquez et al. 23] did in their work on separation of variables of the $\mathrm{SU}(2)$ Calogero-Gaudin system.

In the present case, the true Hamiltonians are not $\mathcal{H}_{j}$ 's but $H_{j}$ 's. We have to rewrite the extra terms on the right hand side of (64), too, as a function of the spectral Darboux coordinates. This is another problem that we have to solve.

To this end, let us note that the defining equation (52) of $\mu_{j}$, which can be rewritten as

$$
\mu_{k}=\sum_{j=1}^{N} \zeta\left(\lambda_{k}-t_{j}\right) \frac{x_{j} \xi_{j}}{2}+p
$$

may be thought of as a system of linear equations for $p$ and $x_{j} \xi_{j} / 2$. If one can solve these equations for $p$ and $x_{j} \xi_{j} / 2$, the solution should be an expression of $p$ and $x_{j} \xi_{j} / 2$ as a function of the spectral Darboux coordinates. Remarkably, these linear equations have the same coefficients as the foregoing linear equations for $\mathcal{H}_{0}$ and $\mathcal{H}_{j}$. Moreover, $x_{j} \xi_{j} / 2$ obey the linear constraint (57), in perfect analogy with the linear constraint (50) for $\mathcal{H}_{j}$.

Thus the two problems, one for $\mathcal{H}_{j}$ and the other for the extra terms in (64), can be reduced to a single problem, namely, solving a system of linear equations of the form

$$
\begin{aligned}
\sum_{j=1}^{N} \zeta\left(\lambda_{k}-t_{j}\right) X_{j}+X_{0} & =b_{k} \quad(k=1, \ldots, N), \\
\sum_{j=1}^{N} X_{j} & =0
\end{aligned}
$$

As we shall show below, this system of linear equations has a unique and explicit solution.

\section{V.2 Solution of linear equations}

We assume that $q \not \equiv 0 \bmod 2 \omega_{1} \mathbf{Z}+2 \omega_{3} \mathbf{Z}$ or, equivalently, $\sigma(q) \neq 0$. The following ensures the uniqueness of solution of (65). 
Lemma 1 If $b_{j}=\cdots=b_{N}=0$, then $X_{0}=X_{1}=\cdots=X_{N}=0$.

Proof. Consider the function

$$
f(z)=\sum_{j=1}^{N} \zeta\left(z-t_{j}\right) X_{j}+X_{0} .
$$

The first $N$ equations of (65) imply that $f(z)$ has zeros at $z=\lambda_{1}, \ldots, \lambda_{N}$. The remaining one ensures that $f(z)$ is a doubly periodic meromorphic function on the $z$-plane. All possible poles are obviously simple and confined to $z=t_{1}, \ldots, t_{N}$ and their translations by the period lattice. Therefore, if $f(z)$ is not identically zero, the zeros $\lambda_{j}$ and the poles $t_{j}$ are constrained as

$$
\sum_{j=1}^{N} \lambda_{j}-\sum_{j=1}^{N} t_{j} \equiv 0 \quad \bmod 2 \omega_{1} \mathbf{Z}+2 \omega_{3} \mathbf{Z},
$$

but this contradicts the assumption that $q \not \equiv 0$; recall the constraint (55) . Thus $f(z)$ is identically zero, and all the coefficients $X_{0}, X_{1}, \cdots, X_{N}$ have to be zero. Q.E.D.

Having proven the uniqueness, the problem is to find a solution by any means. This can be done with the aid of an elliptic analogue of Lagrange's interpolation formula (see Appendix A).

Lemma 2 A solution of (65) is given by

$$
\begin{aligned}
& X_{j}=\sum_{k=1}^{N} \frac{Q\left(t_{j}\right) P\left(\lambda_{k}\right) \sigma\left(t_{j}-\lambda_{k}+q\right) b_{k}}{P^{\prime}\left(t_{j}\right) Q^{\prime}\left(\lambda_{k}\right) \sigma\left(t_{j}-\lambda_{k}\right) \sigma(q)} \\
& X_{0}=-\sum_{j, k=1}^{N} \frac{Q\left(t_{j}\right) P\left(\lambda_{k}\right) \sigma\left(t_{j}-\lambda_{k}+q\right) \zeta\left(\lambda_{k}-t_{j}-q\right) b_{k}}{P^{\prime}\left(t_{j}\right) Q^{\prime}\left(\lambda_{k}\right) \sigma\left(t_{j}-\lambda_{k}\right) \sigma(q)} .
\end{aligned}
$$

Proof. We have only to confirm that these $X_{j}$ and $X_{0}$ do satisfy (65). The last equation of (65) is indeed satisfied as (A.1) shows. As regards the other equations of (65), the main task is to calculate

$$
\sum_{j=1}^{N} \zeta\left(\lambda_{l}-t_{j}\right) X_{j}=\sum_{k=1}^{N}\left(\sum_{j=1}^{N} \frac{Q\left(t_{j}\right) \sigma\left(t_{j}-\lambda_{k}+q\right)}{P^{\prime}\left(t_{j}\right) \sigma\left(t_{j}-\lambda_{k}\right)} \zeta\left(\lambda_{l}-t_{j}\right)\right) \frac{P\left(\lambda_{k}\right) b_{k}}{Q^{\prime}\left(\lambda_{k}\right) \sigma(q)} .
$$

We can use the two identities (A.3) and (A.4) to rewrite the sum over $j$ on the right hand side, and find that

$$
\begin{aligned}
\sum_{j=1}^{N} \zeta\left(\lambda_{l}-t_{j}\right) X_{j} & =\sum_{k, j=1}^{N} \frac{Q\left(t_{j}\right) P\left(\lambda_{k}\right) \sigma\left(t_{j}-\lambda_{k}+q\right) \zeta\left(\lambda_{k}-t_{j}-q\right) b_{k}}{P^{\prime}\left(t_{j}\right) Q^{\prime}\left(\lambda_{k}\right) \sigma\left(t_{j}-\lambda_{k}\right) \sigma(q)}+b_{l} \\
& =-X_{0}+b_{l},
\end{aligned}
$$


which is nothing but the first $N$ equations of (65). Q.E.D.

\section{V.3 Writing $H_{j}$ explicitly}

Let us apply the foregoing formulae (66) and (67) of solution of (65) to the problem of deriving an explicit form of $H_{j}$ as a function of the spectral Darboux coordinates.

If we use the formulae to the case where

$$
X_{j}=\mathcal{H}_{j}, \quad X_{0}=\mathcal{H}_{0}, \quad b_{k}=\mu_{k}^{2}-\sum_{l=1}^{N} C_{l} \wp\left(\lambda_{k}-t_{l}\right),
$$

we find the following expression of $\mathcal{H}_{j}$ and $\mathcal{H}_{0}$ :

$$
\begin{aligned}
\mathcal{H}_{j} & =\sum_{j=1}^{N} \frac{Q\left(t_{j}\right) P\left(\lambda_{k}\right) \sigma\left(t_{j}-\lambda_{k}+q\right)}{P^{\prime}\left(t_{j}\right) Q^{\prime}\left(\lambda_{k}\right) \sigma\left(t_{j}-\lambda_{k}\right) \sigma(q)}\left(\mu_{k}^{2}-\sum_{l=1}^{N} C_{l} \wp\left(\lambda_{k}-t_{l}\right)\right), \\
\mathcal{H}_{0} & =-\sum_{j, k=1}^{N} \frac{Q\left(t_{j}\right) P\left(\lambda_{k}\right) \sigma\left(t_{j}-\lambda_{k}+q\right) \zeta\left(\lambda_{k}-t_{j}-q\right)}{P^{\prime}\left(t_{j}\right) Q^{\prime}\left(\lambda_{k}\right) \sigma\left(t_{j}-\lambda_{k}\right) \sigma(q)}\left(\mu_{k}^{2}-\sum_{l=1}^{N} C_{l} \wp\left(\lambda_{k}-t_{l}\right)\right) .
\end{aligned}
$$

Similarly, if we use the formulae in the case where

$$
X_{j}=\frac{1}{2} x_{j} \xi_{j}, \quad X_{0}=p, \quad b_{k}=\mu_{k}
$$

we find the following expression of $x_{j} \xi_{j} / 2$ and $p$ as a function of the spectral Darboux coordinates:

$$
\begin{aligned}
\frac{1}{2} x_{j} \xi_{j} & =\sum_{k=1}^{N} \frac{Q\left(t_{j}\right) P\left(\lambda_{k}\right) \sigma\left(t_{j}-\lambda_{k}+q\right) \mu_{k}}{P^{\prime}\left(t_{j}\right) Q^{\prime}\left(\lambda_{k}\right) \sigma\left(t_{j}-\lambda_{k}\right) \sigma(q)} \\
p & =-\sum_{j, k=1}^{N} \frac{Q\left(t_{j}\right) P\left(\lambda_{k}\right) \sigma\left(t_{j}-\lambda_{k}+q\right) \zeta\left(\lambda_{k}-t_{j}-q\right) \mu_{k}}{P^{\prime}\left(t_{j}\right) Q^{\prime}\left(\lambda_{k}\right) \sigma\left(t_{j}-\lambda_{k}\right) \sigma(q)} .
\end{aligned}
$$

Thus we have been able to rewrite each term on the right hand side of (64) to an explicit function of the spectral Darboux coordinates.

Although the extra terms on the right hand side of (64) still appear to be in disorder, one can see by but straightforward calculations (see Appendix B) that the sum of these terms boils down to a form similar to the foregoing expression of $\mathcal{H}_{j}$ :

$$
\begin{aligned}
p & -\frac{1}{2} x_{j} \xi_{j}\left(\sum_{k=1}^{N} \zeta\left(t_{j}-\lambda_{k}\right)-\sum_{k \neq j} \zeta\left(t_{j}-t_{k}\right)\right)+\frac{1}{2} \sum_{k \neq j} \zeta\left(t_{j}-t_{k}\right) x_{k} \xi_{k} \\
= & \sum_{k=1}^{N} \frac{Q\left(t_{j}\right) P\left(\lambda_{k}\right) \sigma\left(t_{j}-\lambda_{k}+q\right)}{P^{\prime}\left(t_{j}\right) Q^{\prime}\left(\lambda_{k}\right) \sigma\left(t_{j}-\lambda_{k}\right)}\left(\zeta\left(t_{j}-\lambda_{k}+q\right)-\zeta\left(t_{j}-\lambda_{k}\right)\right) \mu_{k} .
\end{aligned}
$$


Combining these results, we obtain the following expression of $H_{j}$ in terms of the spectral Darboux coordinates:

$$
\begin{aligned}
H_{j}= & \sum_{k=1}^{N} \frac{Q\left(t_{j}\right) P\left(\lambda_{k}\right) \sigma\left(t_{j}-\lambda_{k}+q\right)}{P^{\prime}\left(t_{j}\right) Q^{\prime}\left(\lambda_{k}\right) \sigma\left(t_{j}-\lambda_{k}\right) \sigma(q)} \times \\
& \times\left(\mu_{k}^{2}+\left(\zeta\left(t_{j}-\lambda_{k}+q\right)-\zeta\left(t_{j}-\lambda_{k}\right)\right) \mu_{k}-\sum_{l=1}^{N} C_{l} \wp\left(\lambda_{k}-t_{l}\right)\right) .
\end{aligned}
$$

Eliminating $q$ by (55), we eventually obtain a final expression of the Hamiltonians:

$$
\begin{aligned}
H_{j}= & \sum_{k=1}^{N} \frac{Q\left(t_{j}\right) P\left(\lambda_{k}\right) \sigma\left(\sum_{l \neq k} \lambda_{l}-\sum_{l \neq j} t_{l}\right)}{P^{\prime}\left(t_{j}\right) Q^{\prime}\left(\lambda_{k}\right) \sigma\left(t_{j}-\lambda_{k}\right) \sigma\left(\sum_{l=1}^{N} \lambda_{l}-\sum_{l=1}^{N} t_{l}\right)} \times \\
& \times\left(\mu_{k}^{2}+\left(\zeta\left(t_{j}-\lambda_{k}+q\right)-\zeta\left(t_{j}-\lambda_{k}\right)\right) \mu_{k}-\sum_{l=1}^{N} C_{l} \wp\left(\lambda_{k}-t_{l}\right)\right) .
\end{aligned}
$$

In summary, we have proven the following.

Theorem 1 The isomonodromic $\mathrm{SU}(2)$ Calogero-Gaudin system can be converted to the non-autonomous Hamiltonian system

$$
\frac{\partial \lambda_{k}}{\partial t_{j}}=\frac{\partial H_{j}}{\partial \mu_{k}}, \quad \frac{\partial \mu_{k}}{\partial t_{j}}=-\frac{\partial H_{j}}{\partial \lambda_{j}}
$$

in the spectral Darboux coordinates $\lambda_{j}, \mu_{j}$. The Hamiltonians are given by (74).

\section{Relation to second order scalar ODE}

\section{VI.1 Deriving second order ODE}

The structure of the Hamiltonians $H_{j}$ is very similar to Okamoto's Hamiltonians for isomonodromic deformations of a scalar ODE on a torus [8]. This is not a coincidence, but can be explained in the same way as the case of the $2 \times 2$ Schlesinger system 6 .

A clue is the fact that any $2 \times 2$ matrix system

$$
\frac{d Y}{d z}=L(z) Y, \quad Y=\left(\begin{array}{c}
y_{1} \\
y_{2}
\end{array}\right)
$$

yields a second order scalar ODE of the form

$$
\frac{d^{2} y_{1}}{d z^{2}}+p_{1}(z) \frac{d y_{1}}{d z}+p_{2}(z) y_{1}=0 .
$$


The coefficients $p_{1}(z)$ and $p_{2}(z)$ of the latter are determined by the matrix $L(z)$ as follows:

$$
\begin{aligned}
& p_{1}(z)=-\operatorname{Tr} L(z)-\left(\log L_{12}(z)\right)^{\prime} \\
& p_{2}(z)=\operatorname{det} L(z)-L_{11}^{\prime}(z)+L_{11}(z)\left(\log L_{12}(z)\right)^{\prime} .
\end{aligned}
$$

In our case, $L(z)$ is trace-free, so that the foregoing formulae of $p_{1}(z)$ and $p_{2}(z)$ become slightly simpler: Firstly, $p_{1}(z)$ can be written explicitly as

$$
p_{1}(z)=-\left(\log L_{12}(z)\right)^{\prime}=-\sum_{j=1}^{N} \zeta\left(z-\lambda_{j}\right)+\sum_{j=1}^{N} \zeta\left(z-t_{j}\right),
$$

which implies that $p_{1}(z)$ is doubly periodic. Secondly, $p_{2}(z)$ is also doubly periodic (as the quasi-periodicity of the matrix elements of $L(z)$ implies), and given by the formula

$$
p_{2}(z)=-\frac{1}{2} \operatorname{Tr} L(z)^{2}-L_{11}^{\prime}(z)+L_{11}(z)\left(\log L_{12}(z)\right)^{\prime} .
$$

One can see from this formula that $p_{2}(z)$ has simple poles at $z=\lambda_{j}$ and double poles at $z=t_{j}$. Let us express $p_{2}(z)$ as

$$
p_{2}(z)=\sum_{j=1}^{N} \alpha_{j} \wp\left(z-t_{j}\right)+\sum_{j=1}^{N} \beta_{j} \zeta\left(z-t_{j}\right)+\sum_{j=1}^{N} \gamma_{j} \zeta\left(z-\lambda_{j}\right)+\delta
$$

and determine the coefficients by Laurent expansion at the poles.

1. The first coefficient $\alpha_{j}$ can be read off from the $\left(z-t_{j}\right)^{-2}$ term of the Laurent expansion of $-\operatorname{Tr} L(z)^{2} / 2$ :

$$
\alpha_{j}=-C_{j}=-\operatorname{Tr} A_{j}^{2} / 2
$$

2. The second coefficient $\beta_{j}$ is the residue of $p_{2}(z)$ at $z=t_{j}$. The term $L_{11}^{\prime}(z)$ does not contribute to the residue. The residue of the other terms at $z=t_{j}$ can be expressed as

$$
-\operatorname{Res}_{z=t_{j}} \frac{1}{2} \operatorname{Tr} L(z)^{2}=-\mathcal{H}_{j}
$$

and

$$
\begin{aligned}
\operatorname{Res}_{z=t_{j}} L_{11}(z)\left(\log L_{12}(z)\right)^{\prime}= & -p-\frac{1}{2} \sum_{k \neq j} \zeta\left(t_{j}-t_{k}\right) x_{k} \xi_{k} \\
& +\frac{1}{2} x_{j} \xi_{j}\left(\sum_{k=1}^{N} \zeta\left(t_{j}-\lambda_{k}\right)-\sum_{k \neq j} \zeta\left(t_{j}-t_{k}\right)\right) .
\end{aligned}
$$


As (64) shows, the sum of these two quantities is equal to $-H_{j}$. Therefore

$$
\beta_{j}=\operatorname{Res}_{z=t_{j}} p_{2}(z)=-H_{j}
$$

3. The thrid coefficient $\gamma_{j}$ is the residue of $p_{2}(z)$ at $z=\lambda_{j}$. Since

$$
-\operatorname{Res}_{z=\lambda_{j}} \frac{1}{2} \operatorname{Tr} L(z)^{2}=0
$$

and

$$
\operatorname{Res}_{z=\lambda_{j}} L_{11}(z)\left(\log L_{12}(z)\right)^{\prime}=L_{11}\left(\lambda_{j}\right)=\mu_{j}
$$

$\gamma_{j}$ can be expressed as

$$
\gamma_{j}=\operatorname{Res}_{z=\lambda_{j}} p_{2}(z)=\mu_{j}
$$

Thus the Hamiltonians $H_{j}$ and the "momenta" $\mu_{j}$ can be identified with the residues of $p_{2}(z)$. Exactly the same relation can be seen in the case of Garnier's isomonodromic system on a sphere [6].

\section{VI.2 Another form of second order ODE}

Strictly speaking, however, the second order ODE above differs from that of Okamoto [8] and Kawai [9], who consider a linear ODE of the form

$$
\frac{d^{2} y}{d z^{2}}+p(z) y=0
$$

At least formally, this discrepancy can be removed by the "gauge transformation"

$$
y_{1}=\exp \left(-\frac{1}{2} \int^{z} p_{1}(z) d z\right) y
$$

The coefficient $p(z)$ is given by

$$
p(z)=-\frac{1}{2} p_{1}^{\prime}(z)-\frac{1}{4} p_{1}(z)^{2}+p_{2}(z) .
$$

Note, however, that this is actually a delicate procedure, because the gauge transformation might spoil the isomonodromic property. Fortunately, the present case is free from this problem: The gauge transformation takes the form

$$
y_{1}=\left(\frac{\prod_{j=1}^{N} \sigma\left(z-\lambda_{j}\right)}{\prod_{j=1}^{N} \sigma\left(z-t_{j}\right)}\right)^{1 / 2} y,
$$


and since the factor in front of $y$ has constant monodromy, the isomonodromic property is preserved by the gauge transformation.

The zero-th order term $p(z)$ of the transformed ODE is a doubly periodic meromorphic function with second order poles at $z=\lambda_{j}, t_{j}$. The residues of $p(z)$ at these poles can be readily determined:

$$
\begin{aligned}
& \operatorname{Res}_{z=\lambda_{j}} p(z)=\mu_{j}-2 \sum_{k=1}^{N} \zeta\left(\lambda_{j}-t_{k}\right)+2 \sum_{k \neq j} \zeta\left(\lambda_{j}-\lambda_{k}\right), \\
& \operatorname{Res}_{z=t_{j}} p(z)=-H_{j}-2 \sum_{k=1}^{N} \zeta\left(t_{j}-\lambda_{k}\right)+2 \sum_{k \neq j} \zeta\left(t_{j}-t_{k}\right) .
\end{aligned}
$$

It is rather these quantities that Okamoto [8] and Kawai [9] use as Hamiltonians and conjugate variables of $\lambda_{j}$ 's. We can indeed reformulate our Hamiltonian system in that way. Namely, if we define

$$
\nu_{j}=\operatorname{Res}_{z=\lambda_{j}} p(z), \quad K_{j}=-\operatorname{Res}_{z=t_{j}} p(z),
$$

these quantities satisfy the equation

$$
\sum_{j=1}^{N} \mu_{j} d \lambda_{j}-\sum_{j=1}^{N} H_{j} d t_{j}=\sum_{j=1}^{N} \nu_{j} d \lambda_{j}-\sum_{j=1}^{N} K_{j} d t_{j}+\text { exact form. }
$$

This implies that $\lambda_{j}$ 's and $\nu_{j}$ 's are a new set of Darboux coordinates, and that the previous Hamiltonian system is now converted to the new Hamiltonian system

$$
\frac{\partial \lambda_{k}}{\partial t_{j}}=\frac{\partial K_{j}}{\partial \nu_{k}}, \quad \frac{\partial \nu_{k}}{\partial t_{j}}=-\frac{\partial K_{j}}{\partial \lambda_{j}}
$$

\section{VI.3 Reconstructing $2 \times 2$ matrix system}

Let us now consider the inverse problem. Namely, given the isomonodromic deformations of the second oder scalar ODE above, the problem is to reconstruct a $2 \times 2$ matrix system. A similar problem is discussed by Okamoto [6, Section 3] in the case of isomonodromic deformations on a sphere. In our case, the presence of diagonal gauge transformations (19) allows us to fix the coefficient $\kappa$ as $\kappa=1$. Therefore we have only to show how to reconstruct the dynamical variables $q, p, A_{k}$ 's of the matrix system from $\lambda_{j}, \mu_{j}$ of the scalar ODE.

One can indeed reconstruct the $L$-matrix $L(z)$ by an algebraic procedure as follows. The first step is to reconsider (53) and (55) as definition of $L_{12}(z)$ and $q$. The coefficient $\kappa$ 
is chosen to be $\kappa=1$ as remarked above. $A_{j}^{-}$'s are thus determined. Secondly, let $L_{11}(z)$ be a function of the form

$$
L_{11}(z)=p+\sum_{j=1}^{N} \phi\left(q, z-t_{j}\right) A_{j}^{3}
$$

that satisfy the interpolation relations $L_{11}\left(\lambda_{j}\right)=\mu_{j}$ for $j=1, \ldots, N$. As we have seen in Section $\mathrm{V}$, these relations can be solved for $A_{j}^{3}$ and $p$ under the constraint $\sum_{j=1}^{N} A_{j}^{3}=0$. As (6) suggests, $A_{j}^{+}$'s are to be determined as

$$
A_{j}^{+}=\frac{\theta_{j}^{2} / 4-\left(A_{j}^{3}\right)^{2}}{A_{j}^{-}} .
$$

One can thus reconstruct $L(z)$ from the scalar ODE.

\section{Conclusion}

We have applied the method of spectral Darboux coordinates to Korotkin and Samtleben's isomonodromic system on a torus [10]. The isomonodromic system has been thus converted to a non-autonomous Hamiltonian system in the spectral Darboux coordinates. Although the Hamiltonians turn out to be a considerably intricate function, the method we have used is a rather straightforward analogue of the usual method for isomonodromic deformations on a sphere.

Our non-autonomous Hamiltonian system may be thought of as an elliptic analogue of Garnier's isomonodromic systems [2, 4, 6]. Almost the same system has been derived by Okamoto from isomonodromic deformations of a second order scalar ODE on a torus [8]. We have seen how these two systems are related. Speaking differently, our approach from a $2 \times 2$ matrix system reveals a hidden algebro-geometric meaning of the Hamiltonian structure in Okamoto's work [6].

An important lesson of the present work is that the notions of spectral curve and spectral Darboux coordinates persist to be useful and essential beyond isospectral deformations. This observation lies in the heart of the work of Harnad and Wisse [18. We have confirmed it for an example of isomonodromic deformations on a torus.

In this respect, an interesting problem is to describe the isomonodromic $\mathrm{SU}(2)$ pure Gaudin system [12, 14] from the same point of view. Separation of variables of the 
isospectral partner has been studied by Sklyanin and Takebe [28] (see also the paper of Hurtubise and Kjiri [29] for geometric aspects). The work of Sklyanin and Takebe shows that separation of variables of this system is technically far more complicated than the Calogero-Gaudin system. This will be also the case for the isomonodromic analogue.

Let us conclude the present consideration with a remark on trigonometric and rational analogues. The trigonometric and rational analogues of Korotkin and Samtleben's isomonodromic deformations can be obtained by replacing the basic functions $\sigma(z), \zeta(z)$ and $\phi(u, z)$ by the following trigonometric or rational functions:

1. Trigonometric model

$$
\sigma(z)=\sin z, \quad \zeta(z)=\frac{\cos z}{\sin z}, \quad \phi(u, z)=\frac{\cos z}{\sin z}-\frac{\cos u}{\sin u} .
$$

2. Rational model

$$
\sigma(z)=z, \quad \zeta(z)=\frac{1}{z}, \quad \phi(u, z)=\frac{1}{z}-\frac{1}{u} .
$$

A hyperbolic model will be obtained if one replaces the trigonometric functions by the corresponding hyperbolic functions. These are nothing but the well known pattern of degeneration of the Calogero-Moser systems; the Calogero-Gaudin systems, too, obey this pattern. In fact, it is the rational model in this list that Brzeziński considered in his work 22]. One can formulate an isomonodromic partner of these degenerate CalogeroGaudin systems as in the case of the elliptic model. Presumably, those isomonodromic systems will not be known in the literature.

\section{Acknowledgements}

I am grateful to Shingo Kawai and Takashi Takebe for many useful comments. This work is partially supported by the Grant-in-Aid for Scientific Research (No. 12640169) from the Ministry of Education, Culture, Sports and Technology.

\section{A Interpolation formula}

Let us examine the auxiliary function

$$
f_{k}(z)=\frac{Q(z) \sigma\left(z-\lambda_{k}+q\right)}{P(z) \sigma\left(z-\lambda_{k}\right)} .
$$


This is a doubly periodic meromorphic function with simple zeros at $\lambda_{j}(j \neq k)$ and $\lambda_{k}-q$ and simple poles at $t_{j}(j=1, \ldots, N)$. By the residue theorem, the residues

$$
\operatorname{Res}_{z=t_{j}} f_{k}(z)=\frac{Q\left(t_{j}\right) \sigma\left(t_{j}-\lambda_{k}+q\right)}{P^{\prime}\left(t_{j}\right) \sigma\left(t_{j}-\lambda_{k}\right)}
$$

at the poles $z=t_{j}$ obey the sum-to-zero constraint

$$
\sum_{j=1}^{N} \frac{Q\left(t_{j}\right) \sigma\left(t_{j}-\lambda_{k}+q\right)}{P^{\prime}\left(t_{j}\right) \sigma\left(t_{j}-\lambda_{k}\right)}=0
$$

Let us consider the linear combination

$$
\sum_{j=1}^{N} \frac{Q\left(t_{j}\right) \sigma\left(t_{j}-\lambda_{k}+q\right)}{P^{\prime}\left(t_{j}\right) \sigma\left(t_{j}-\lambda_{k}\right)} \zeta\left(z-t_{j}\right)
$$

of $\zeta\left(z-t_{j}\right)$ weighted by these residues. Since this function is a doubly periodic meromorphic function with the same set of simple poles and residues as $f_{k}(z)$, it differs from $f_{k}(z)$ by at most a constant:

$$
\frac{Q(z) \sigma\left(z-\lambda_{k}+q\right)}{P(z) \sigma\left(z-\lambda_{k}\right)}=\sum_{j=1}^{N} \frac{Q\left(t_{j}\right) \sigma\left(t_{j}-\lambda_{k}+q\right)}{P^{\prime}\left(t_{j}\right) \sigma\left(t_{j}-\lambda_{k}\right)} \zeta\left(z-t_{j}\right)+\text { constant. }
$$

Moreover, since the left hand side vanishes at $z=\lambda_{k}-q$, the constant term on the right hand side can be easily determined as follows:

$$
\text { constant }=-\sum_{j=1}^{N} \frac{Q\left(t_{j}\right) \sigma\left(t_{j}-\lambda_{k}+q\right)}{P^{\prime}\left(t_{j}\right) \sigma\left(t_{j}-\lambda_{k}\right)} \zeta\left(\lambda_{k}-t_{j}-q\right) .
$$

We thus obtain the interpolation formula

$$
\frac{Q(z) \sigma\left(z-\lambda_{k}+q\right)}{P(z) \sigma\left(z-\lambda_{k}\right)}=\sum_{j=1}^{N} \frac{Q\left(t_{j}\right) \sigma\left(t_{j}-\lambda_{k}+q\right)}{P^{\prime}\left(t_{j}\right) \sigma\left(t_{j}-\lambda_{k}\right)}\left(\zeta\left(z-t_{j}\right)-\zeta\left(\lambda_{k}-t_{j}-q\right)\right) .
$$

One can derive the following three identities from this interpolation formula.

1. Since the left hand side of the interpolation formula vanishes at $z=\lambda_{l}(l \neq k)$,

$$
\sum_{j=1}^{N} \frac{Q\left(t_{j}\right) \sigma\left(t_{j}-\lambda_{k}+q\right)}{P^{\prime}\left(t_{j}\right) \sigma\left(t_{j}-\lambda_{k}\right)}\left(\zeta\left(\lambda_{l}-t_{j}\right)-\zeta\left(\lambda_{k}-t_{j}-q\right)\right)=0 \quad(l \neq k) .
$$

2. By letting $z \rightarrow \lambda_{k}$ in the interpolation formula,

$$
\sum_{j=1}^{N} \frac{Q\left(t_{j}\right) \sigma\left(t_{j}-\lambda_{k}+q\right)}{P^{\prime}\left(t_{j}\right) \sigma\left(t_{j}-\lambda_{k}\right)}\left(\zeta\left(\lambda_{k}-t_{j}\right)-\zeta\left(\lambda_{k}-t_{j}-q\right)\right)=\frac{Q^{\prime}\left(\lambda_{k}\right) \sigma(q)}{P^{\prime}\left(\lambda_{k}\right)}
$$


3. By replacing $k \rightarrow l, j \rightarrow k$ and separating a term from the sum, the interpolation formula takes the form

$$
\begin{aligned}
\sum_{k \neq j} & \frac{Q\left(t_{k}\right) \sigma\left(t_{k}-\lambda_{l}+q\right)}{P^{\prime}\left(t_{k}\right) \sigma\left(t_{k}-\lambda_{l}\right)} \zeta\left(z-t_{k}\right) \\
= & \frac{Q(z) \sigma\left(z-\lambda_{l}+q\right)}{P(z) \sigma\left(z-\lambda_{l}\right)}-\frac{Q\left(t_{j}\right) \sigma\left(t_{j}-\lambda_{l}+q\right)}{P^{\prime}\left(t_{j}\right) \sigma\left(t_{j}-\lambda_{l}\right)} \zeta\left(z-t_{j}\right) \\
& +\sum_{k=1}^{N} \frac{Q\left(t_{k}\right) \sigma\left(t_{k}-\lambda_{l}+q\right)}{P^{\prime}\left(t_{k}\right) \sigma\left(t_{k}-\lambda_{l}\right)} \zeta\left(\lambda_{l}-t_{k}-q\right)
\end{aligned}
$$

By letting $z \rightarrow t_{j}$

$$
\begin{aligned}
\sum_{k \neq j} & \frac{Q\left(t_{k}\right) \sigma\left(t_{k}-\lambda_{l}+q\right)}{P^{\prime}\left(t_{k}\right) \sigma\left(t_{k}-\lambda_{l}\right)} \zeta\left(t_{j}-t_{k}\right) \\
= & \frac{Q\left(t_{j}\right) \sigma\left(t_{j}-\lambda_{l}+q\right)}{P^{\prime}\left(t_{j}\right) \sigma\left(t_{j}-\lambda_{\ell}\right)}\left(-\frac{1}{2} \frac{P^{\prime \prime}\left(t_{j}\right)}{P^{\prime}\left(t_{j}\right)}+\frac{Q^{\prime}\left(t_{j}\right)}{Q\left(t_{j}\right)}-\zeta\left(t_{j}-\lambda_{l}\right)+\zeta\left(t_{j}-\lambda_{l}+q\right)\right) \\
& +\sum_{k=1}^{N} \frac{Q\left(t_{k}\right) \sigma\left(t_{k}-\lambda_{l}+q\right)}{P^{\prime}\left(t_{k}\right) \sigma\left(t_{k}-\lambda_{l}\right)} \zeta\left(\lambda_{l}-t_{k}-q\right) .
\end{aligned}
$$

\section{B Calculation of extra terms in (64)}

Let us use (70) to rewrite the last piece on the right hand side of (64) as

$$
\frac{1}{2} \sum_{k=1}^{N} \zeta\left(t_{j}-t_{k}\right) x_{k} \xi_{k}=\sum_{l=1}^{N}\left(\sum_{k \neq j} \frac{Q\left(t_{k}\right) \sigma\left(t_{k}-\lambda_{l}+q\right)}{P^{\prime}\left(t_{k}\right) \sigma\left(t_{k}-\lambda_{l}\right)} \zeta\left(t_{j}-t_{k}\right)\right) \frac{P\left(\lambda_{l}\right) \mu_{l}}{Q^{\prime}\left(\lambda_{l}\right) \sigma(q)} .
$$

The sum over $k \neq j$ arising here has been partially calculated in (A.5). Using the identities

$$
\frac{1}{2} \frac{P^{\prime \prime}\left(t_{j}\right)}{P^{\prime}\left(t_{j}\right)}=\sum_{k \neq j} \zeta\left(t_{j}-t_{k}\right), \quad \frac{Q^{\prime}\left(t_{j}\right)}{Q\left(t_{j}\right)}=\sum_{k=1}^{N} \zeta\left(t_{j}-\lambda_{k}\right)
$$

on the right hand side of (A.5), one can rewrite the foregoing quantity as

$$
\begin{aligned}
& \frac{1}{2} \sum_{k=1}^{N} \zeta\left(t_{j}-t_{k}\right) x_{k} \xi_{k} \\
& =\sum_{l=1}^{N} \frac{Q\left(t_{j}\right) P\left(\lambda_{l}\right) \sigma\left(t_{k}-\lambda_{l}+q\right) \mu_{\ell}}{P^{\prime}\left(t_{j}\right) Q^{\prime}\left(\lambda_{l}\right) \sigma\left(t_{j}-\lambda_{l}\right) \sigma(q)}\left(-\sum_{k \neq j} \zeta\left(t_{j}-t_{k}\right)+\sum_{k=1}^{N} \zeta\left(t_{j}-\lambda_{k}\right)\right) \\
& \quad+\sum_{l=1}^{N} \frac{Q\left(t_{j}\right) P\left(\lambda_{l}\right) \sigma\left(t_{k}-\lambda_{l}+q\right)}{P^{\prime}\left(t_{j}\right) Q^{\prime}\left(\lambda_{l}\right) \sigma\left(t_{j}-\lambda_{l}\right) \sigma(q)}\left(\zeta\left(t_{j}-\lambda_{l}+q\right)-\zeta\left(t_{j}-\lambda_{l}\right)\right) \mu_{\ell} \\
& \quad+\sum_{k, l=1}^{N} \frac{Q\left(t_{k}\right) P\left(\lambda_{l}\right) \sigma\left(t_{k}-\lambda_{l}+q\right) \zeta\left(\lambda_{l}-t_{k}-q\right) \mu_{l}}{P^{\prime}\left(t_{k}\right) Q^{\prime}\left(\lambda_{l}\right) \sigma\left(t_{k}-\lambda_{l}\right) \sigma(q)} .
\end{aligned}
$$


By (70) and (71), the first and third lines on the right hand side turn into the following form:

$$
\begin{aligned}
\text { first line } & =-\frac{1}{2} x_{j} \xi_{j}\left(\sum_{k \neq j} \zeta\left(t_{j}-t_{k}\right)-\sum_{k=1}^{N} \zeta\left(t_{j}-\lambda_{k}\right)\right), \\
\text { third line } & =-p .
\end{aligned}
$$

Since the sum of these two cancels the second and third pieces on the right hand side of (64), we eventually obtain the identity

$$
\begin{aligned}
p & -\frac{1}{2} x_{j} \xi_{j}\left(\sum_{k=1}^{N} \zeta\left(t_{j}-\lambda_{k}\right)-\sum_{k \neq j} \zeta\left(t_{j}-t_{k}\right)\right)+\frac{1}{2} \sum_{k \neq j} \zeta\left(t_{j}-t_{k}\right) x_{k} \xi_{k} \\
= & \sum_{l=1}^{N} \frac{Q\left(t_{j}\right) P\left(\lambda_{l}\right) \sigma\left(t_{j}-\lambda_{l}+q\right)}{P^{\prime}\left(t_{j}\right) Q^{\prime}\left(\lambda_{l}\right) \sigma\left(t_{j}-\lambda_{l}\right) \sigma(q)}\left(\zeta\left(t_{j}-\lambda_{l}+q\right)-\zeta\left(t_{j}-\lambda_{l}\right)\right) \mu_{l},
\end{aligned}
$$

which is nothing but (72).

\section{Zero-curvature equations in more detail}

Since the diagonal part of the zero-curvature equation (37) has been specified in Section III, let us now examine the off-diagonal part.

As regards the upper right part, the matrix element of the commutator $\left[\widetilde{M}_{j}(z), \widetilde{M}_{k}(z)\right]$ reads

$$
\begin{aligned}
{\left[\widetilde{M}_{j}(z), \widetilde{M}_{k}(z)\right]_{12}=} & 2\left(p_{j}+\zeta\left(z-t_{j}\right) A_{j}^{3}\right) \phi\left(q, z-t_{k}\right) A_{k}^{-} \\
& -2 \phi\left(q, z-t_{j}\right) A_{j}^{-}\left(p_{k}+\zeta\left(z-t_{k}\right) A_{k}^{3}\right) .
\end{aligned}
$$

One can use the functional identity (11) in the form

$$
\phi(u, z-w) \zeta(z)=-\phi(u, z) \phi(u,-w)+\phi(u, z-w) \zeta(w)-\phi_{u}(u, z-w)
$$

to eliminate $\zeta\left(z-t_{j}\right) \phi\left(q, z-t_{k}\right)$ and $\phi\left(q, z-t_{j}\right) \zeta\left(z-t_{k}\right)$ as

$$
\begin{aligned}
{\left[\widetilde{M}_{j}(z), \widetilde{M}_{k}(z)\right]_{12}=} & 2 \phi\left(q, z-t_{k}\right)\left(p_{j} A_{k}^{-}+\zeta\left(t_{k}-t_{j}\right) A_{j}^{3} A_{k}^{-}+\phi\left(q, t_{k}-t_{j}\right) A_{j}^{-} A_{k}^{3}\right) \\
& -2 \phi\left(q, z-t_{j}\right)\left(p_{k} A_{j}^{-}+\zeta\left(t_{j}-t_{k}\right) A_{j}^{-} A_{k}^{3}+\phi\left(q, t_{j}-t_{k}\right) A_{j}^{3} A_{k}^{-}\right) \\
& -2 \phi_{u}\left(q, z-t_{k}\right) A_{j}^{3} A_{k}^{-}+2 \phi_{u}\left(q, z-t_{j}\right) A_{j}^{-} A_{k}^{3} .
\end{aligned}
$$


On the other hand, the derivative part of the zero-curvature equation can be expressed as

$$
\begin{aligned}
& \frac{\partial \widetilde{M}_{k, 12}(z)}{\partial t_{j}}-\frac{\partial \widetilde{M}_{j, 12}(z)}{\partial t_{k}} \\
& \quad=\phi\left(q, z-t_{k}\right) \frac{\partial A_{k}^{-}}{\partial t_{j}}+\phi_{u}\left(q, z-t_{k}\right) \frac{\partial q}{\partial t_{j}} A_{k}^{-}-\phi\left(q, z-t_{j}\right) \frac{\partial A_{j}^{-}}{\partial t_{k}}-\phi_{u}\left(q, z-t_{j}\right) \frac{\partial q}{\partial t_{k}} A_{j}^{-} .
\end{aligned}
$$

Thus the $\phi_{u}$ terms cancel out in the zero-curvature equation and one is left with an equation of the form

$$
\phi\left(q, z-t_{j}\right)(\cdots)+\phi\left(q, z-t_{k}\right)(\cdots)=0
$$

with the coefficients $(\cdots)$ that do not depend on $z$. As it turns out, these coefficients are exactly the same as some of the equations of motion of $q, A_{j}$ and $A_{k}$. One can thus see that this part of the zero-curvature equations is automatically satisfied.

The lower left part of the zero-curvature equation can be treated in the same way.

\section{Integrability of (38)}

In components, the closedness condition $d \omega=0$ reads

$$
\begin{array}{r}
\partial_{t_{j}}\left(\phi\left(q, t_{j}-t_{k}\right)\left(\zeta\left(t_{j}-t_{k}-q\right)+\zeta(q)\right) A_{j}^{+} A_{k}^{-}\right) \\
+(\text {cyclic permutations of } j, k, \ell)=0 .
\end{array}
$$

The goal is to show that these equations are indeed satisfied under the equations of motion (24), (25), (26) of $q, p$ and $A$ 's. Applying the Leibniz rule to the left hand side of (D.6) yields such terms as

$$
\begin{array}{r}
-\phi\left(q, t_{j}-t_{k}\right)\left(\left(\zeta\left(t_{j}-t_{k}-q\right)+\zeta(q)\right)^{2}+\zeta^{\prime}\left(t_{j}-t_{k}-q\right)+\zeta^{\prime}(q)\right) \frac{\partial q}{\partial t_{j}} A_{j}^{+} A_{k}^{-} \\
+\phi\left(q, t_{j}-t_{k}\right)\left(\zeta\left(t_{j}-t_{k}-q\right)+\zeta(q)\right)\left(\frac{\partial A_{j}^{+}}{\partial t_{j}} A_{k}^{-}+A_{j}^{+} \frac{\partial A_{k}^{-}}{\partial t_{j}}\right)
\end{array}
$$

and their cyclic permutations. One can eliminate the derivatives of $q, p$ and $A$ 's by the equations of motion; this in turn yields linear and quadratic combinations of $\phi$ 's. As regards the quadratic combinations, one can use the functional identity (11) to reduce such terms to a linear combinations of $\phi$ 's, e.g.,

$\phi\left(q, t_{j}-t_{k}\right) \phi\left(q, t_{\ell}-t_{j}\right)=\phi\left(q, t_{\ell}-t_{k}\right)\left(\zeta\left(q-t_{\ell}+t_{k}\right)-\zeta(q)+\zeta\left(t_{j}-t_{k}\right)+\zeta\left(t_{\ell}-t_{j}\right)\right)$, 
etc. After some more algebra, one can thus eventually convert (D.6) to equations of the form

$$
\phi\left(q, t_{j}-t_{k}\right) c_{j k \ell} A_{j}^{+} A_{k}^{-} A_{\ell}^{3}+(\text { cyclic permutations of } j, k, \ell)=0
$$

where

$$
\begin{aligned}
c_{j k \ell}= & \left(\zeta\left(t_{j}-t_{k}-q\right)+\zeta(q)\right)^{2}+\zeta^{\prime}\left(t_{j}-t_{k}-q\right)+\zeta^{\prime}(q) \\
& +\left(\zeta\left(t_{j}-t_{k}-q\right)+\zeta(q)\right)\left(\zeta\left(t_{\ell}-t_{j}\right)-\zeta\left(t_{\ell}-t_{k}\right)\right) \\
& +\left(\zeta\left(t_{\ell}-t_{k}-q\right)+\zeta(q)\right)\left(-\zeta\left(t_{j}-t_{k}-q\right)-\zeta(q)+\zeta\left(t_{\ell}-t_{k}\right)+\zeta\left(t_{j}-t_{\ell}\right)\right) \\
& +\left(\zeta\left(t_{j}-t_{\ell}-q\right)+\zeta(q)\right)\left(-\zeta\left(t_{j}-t_{k}-q\right)-\zeta(q)+\zeta\left(t_{j}-t_{\ell}\right)+\zeta\left(t_{\ell}-t_{k}\right)\right) .
\end{aligned}
$$

Actually, these coefficients $c_{j k \ell}$ turn out to vanish identically. One can indeed verify that the function

$$
\begin{aligned}
f_{j k \ell}(z)= & \left(\zeta\left(t_{j}-t_{k}-z\right)+\zeta(z)\right)^{2}+\zeta^{\prime}\left(t_{j}-t_{k}-z\right)+\zeta^{\prime}(z) \\
& +\left(\zeta\left(t_{j}-t_{k}-z\right)+\zeta(z)\right)\left(\zeta\left(t_{\ell}-t_{j}\right)-\zeta\left(t_{\ell}-t_{k}\right)\right) \\
& +\left(\zeta\left(t_{\ell}-t_{k}-z\right)+\zeta(z)\right)\left(-\zeta\left(t_{j}-t_{k}-z\right)-\zeta(z)+\zeta\left(t_{\ell}-t_{k}\right)+\zeta\left(t_{j}-t_{\ell}\right)\right) \\
& +\left(\zeta\left(t_{j}-t_{\ell}-z\right)+\zeta(z)\right)\left(-\zeta\left(t_{j}-t_{k}-z\right)-\zeta(z)+\zeta\left(t_{j}-t_{\ell}\right)+\zeta\left(t_{\ell}-t_{k}\right)\right)
\end{aligned}
$$

of the complex variable $z$ is a doubly periodic entire function with a zero at $z=\left(t_{j}-t_{k}\right) / 2$; this implies that $f_{j k \ell}(z)=0$. 


\section{References}

[1] R. Fuchs, "Über lineare homogene Differentialgleichungern zweiter Ordnung mit im endlich gelegene wesentlich singulären Stellen," Math. Ann. 63, 301-321 (1907).

[2] R. Garnier, "Sur des équations différentielles du troisième ordre dont l'intégrale est uniform et sur une classe d'équations nouvelles d'ordre supérieur dont l'intégrale générale à ses point critiques fixés," Ann. Sci. École Norm. Sup. (3) 29, 1-126 (1912).

[3] L. Schlesinger, "Über eine Klasse von Differentialsystemen beliebliger Ordnung mit festen kritischer Punkten," J. für Math. 141, 96-145 (1912).

[4] R. Garnier, "Solution du problème de Riemann pour les systèmes différentiels linéaires du second ordre," Ann. Sci. École Norm. Sup. (3) 43, 177-307 (1926).

[5] K. Okamoto, "On Fuchs's problem on a torus, I," Funkcial. Ekvac. 14, 137-152 (1971); "Sur le problème de Fuchs sur un tore, II," J. Fac. Sci. Univ. Tokyo, Sec.

IA 24, 357-372 (1977); "Déformation d'une équation différentielle linéaire avec une singularité irrégulière sur un tore," J. Fac. Sci. Univ. Tokyo, Sec. IA 26, 501-518 (1979); "The Hamiltonian structure derived from the holonomic deformation of the linear ordinary differential equations on an elliptic curve," Sci. Papers, Col. Art. Sci. Univ. Tokyo 37, 1-11 (1987).

[6] K. Okamoto, "Isomonodromic deformation and Painlevé equation, and the Garnier system," J. Fac. Sci. Univ. Tokyo, Sec. IA, 33, 575-618 (1986).

[7] K. Iwasaki, "Moduli and deformation for Fuchsian projective connections on a Riemann surface," J. Fac. Sci. Univ. Tokyo, Sect. IA 38, 431-531 (1991); "Fuchsian moduli on Riemann surface - its Poisson structure and the Poincaré-Lefshetz duality," Pacific J. Math. 155, 319-340 (1992).

[8] K. Okamoto, "On the holonomic deformations of linear ordinary differential equations on an elliptic curve," Kyushu J. Math. 49, 281-308 (1995).

[9] S. Kawai, "Isomonodromic deformation of Fuchsian-type projective connections on elliptic curves," RIMS Kokyuroku vol. 1022, pp.53-57 (RIMS, Kyoto University, 1997). 
[10] D.A. Korotkin and J.A.H. Samtleben, "On the quantization of isomonodromic deformations on the torus," Intern. J. Mod. Phys. A12, 2013-2030 (1997).

[11] A.M. Levin and M.A. Olshanetsky, "Painlevé-Calogero correspondence," in CalogeroMoser-Sutherland models, CRM Ser. Math. Phys., pp. 313-332 (Springer, New York, 2000); "Hierarchies of isomonodromic deformations and Hitchin systems," in Moscow Seminar in Mathematical Physics, Amer. Math. Soc. Transl. Ser. 2, vol. 191, pp. 223262 (American Mathematical Society, Providence, RI, 1999).

[12] K. Takasaki, "Gaudin model, KZ equation, and isomonodromic problem on torus," Lett. Math. Phys. 44, 143-156 (1998).

[13] K. Takasaki, "Elliptic Calogero-Moser systems and isomonodromic deformations," J. Math. Phys. 40, 5787-5821 (1999).

[14] D. Korotkin, N. Manojlović and H. Samtleben, "Schlesinger transformations for elliptic isomonodromic deformations," J. Math. Phys. 41, 3125-3141 (2000).

[15] I. Krichever, Isomonodromic equations on algebraic curves, canonical transformations and Whitham equations, e-print arXiv:hep-th/0112096.

[16] M.R. Adams, J. Harnad and J. Hurtubise, "Darboux coordinates and LiouvilleArnold integration in loop algebras," Commun. Math. Phys. 155, 385-413 (1993).

[17] J. Harnad, "Dual isomonodromic deformations and moment maps to loop algebras," Commun. Math. Phys. 166, 337-365 (1994).

[18] J. Harnad and M.A. Wisse, "Loop algebra moment maps and Hamiltonian models for the Painlevé transcendents," AMS-Fields Inst. Commun. 7, 155-169 (1996).

[19] J. Moser, "Geometry of quadrics and spectral theory," in: W.-Y. Hsiang et al. (eds.), The Chern Symposium 1979, pp. 147-188 (Springer-Verlag, 1980).

[20] N. Nekrasov, "Holomorphic bundles and many body problems," Commun. Math. Phys. 180, 587-603 (1996).

[21] B. Enriquez and V. Rubtsov, "Hitchin systems, higher Gaudin operators and rmatrices," Math. Res. Lett. 3, 343-357 (1996). 
[22] T. Brzeziński, "Dynamical $r$-matrices and separation of variables: the Generalized Calogero-Moser models," Phys. Lett. B325, 401-408 (1994).

[23] B. Enriquez, B. Feigin and V. Rubtsov, "Separation of variables for Gaudin-Calogero systems," Compositio Math. 110, 1-16 (1998).

[24] E.K. Sklyanin, "Separation of variables - new trends," Prog. Theor. Phys. Suppl. 118, 35-60 (1995).

[25] J. Avan and M. Talon, "Classical $R$-matrix structure for the Calogero model," Phys. Lett. B303, 33-37 (1993).

[26] E. Sklyanin, "Dynamical $r$-matrices for the elliptic Calogero-Moser model," Alg. and Anal. 6, 227-237 (1994).

[27] H.W. Braden and T. Suzuki, " $R$-matrices for elliptic Calogero-Moser models," Lett. Math. Phys. 30, 147-158 (1994).

[28] E. Sklyanin and T. Takebe, "Separation of variables in the elliptic Gaudin model," Commun. Math. Phys. 204, 17-38 (1999).

[29] J.C. Hurtubise and M. Kjiri, "Separating coordinates for the generalized Hitchin systems and the classical r-matrix," Commun. Math. Phys. 210 (2000), 521-540. 